\title{
Biotechnology of Woody Environmental Crops
}

\author{
Charles H. Michler \\ U.S. Department of Agriculture Forest Service, North Central Forest Experiment Station, Forestry Sciences \\ Laboratory, P.O. Box 898, Rhinelander, WI 54501
}

Scientists working in woody environmental plant improvement have not taken advantage of new biotechnologies available to them. Such technologies could provide numerous opportunities for rapid genetic improvement.

The meaning of the term biotechnology is evolving; for purposes of simplicity, it will be defined here as the following three processes: in vitro genetic selection, in vitro mutagenesis, and genetic engineering. These processes can be used to select genetically variant or mutant cells or to insert DNA into cells to produce genetically improved plants.

In vitro genetic selection, often termed "somaclonal selection" (Larkin and Scowcroft, 1981), enables one to use techniques to select genetically variant cells and verify genetic variation in regenerated plants by biochemical or molecular techniques. The use of cell and tissue culture selection procedures results in regenerants with genotypic variation that originates from a single genotype. Genetic variation may exist within genetically heterogenous cell cultures either as pre-existing potential for genetic variation or as potential for genetic variability induced by the culture process. Selected cells can be regenerated and resultant variant plants can be tested for stability of the genetic alteration.

Mutagenesis, in the context of plant biotechnology, is a classical means of altering plant genomes by applying either radiation or chemicals, coupled with regeneration of genetically altered plants in vitro. Examples of mutagenic agents are gamma rays, X-rays, ethyl methanesulfonate (EMS), 2,4-dichlorophenoxyacetic acid (2,4-D), and colchicine. Plants that result from use of these mutagens usually have random genetic mutations; the genetic mutations cannot be targeted. The applicability of chemical and physical mutagenesis is limited in biotechnology because lack of targeting results in low selection efficiency for a given target. In addition, many undesirable genetic characteristics may also be obtained by mutagenesis.

Genetic engineering techniques often use recombinant DNA that is inserted into cells by Agrobacterium-mediated transfer, microprojectile bombardment (biolistics), electric current (electroporation), microinjection, and polyethylene glycol-mediated osmotic insertion. Protoplasts may be needed for the latter three techniques to enable DNA to cross the plasmalemma.

Plant regeneration and clonal micropropagation are important requirements for using biotechnologies. These processes use tissue culture techniques to successfully apply biotechnologies to produce genetically altered plants. Plants can be regenerated by shoot organogenesis or somatic embryogenesis and then clonally micropropagated, usually by proliferation of axillary buds from shoot cultures.

Micropropagation has been used successfully for commercial purposes with woody environmental crops such as Betula, Syringa, Malus, Prunes, Pyrus, Kalmia, Rhododendron, Rosa, and Amelanchier spp. (McCown, 1989). Many other species are produced on a smaller scale for profitable niches in national and regional markets.

I will discuss here the extent of biotechnology research with woody environmental crops, progress within those programs, and problems that impede the use or success of biotechnologies. My overview should help to define research needs and opportunities for applying biotechnology to woody environmental plants.

\section{HISTORICAL BACKGROUND}

Genetic improvement programs for woody environmental plants traditionally have not been structured, focused, cohesive attempts to achieve particular genetic traits. For example, many woody en- vironmental plant genotypes have been selected from outplanted seedlings of half-sib families or from bud mutations that were selected and vegetatively propagated. Thus, the genomes of most woody environmental plants have hardly been exploited for genetic improvement; such plants may have specific genetic characteristics that enhance their utility in horticulture but still are basically wild types.

Biotechnology offers opportunities, in addition to conventional selection and breeding, to use existing plant germplasm for genetic improvement, such as by introducing new genes for commercial characteristics. In some cases, in vitro genetic selection is the most rapid means to produce genetic variants with new characteristics because molecular skills and recombinant DNA are not needed. Genetic variants may be selected in vitro, validated in greenhouse tests, and placed into field evaluation in as little as $1 \frac{1}{2}$ to 2 years. Of course, confirmation by breeding would take much longer, depending on the age at which the plant produces flowers.

Plant biotechnologies have been available for plant improvement application in recent years, but successful applications to woody environmental plants have been few. All examples have been variants selected from micropropagated progeny, without genetic proof of the basis for morphological characters. Examples of these variants are compact forms of Rhododendron (McCown, 1989), chimeral reversions of Rhododendron (R.D. Lineberger, personal communication), and variegated foliage of Paulownia tomentosa 'Somaclonal Snowstorm' (Marcotrigiano and Jagannathan, 1988). It has not been reported if any of these selections have had longterm, multigenerational stability of improved traits. Data on vegetative stability and heritability of traits are necessary to evalute if in vitro variants are genetically altered or epigenetic artifacts of the tissue culture process.

Some exciting plant improvements and biotechnological applications have been made to forest trees that could be directly applied to woody environmental plants. These applications are with techniques using in vitro genetic selection and genetic engineering.

In vitro genetic selection has been used to produce putative herbicide- and disease-tolerant trees and trees with leaf variegation. Hybrid poplars were produced that have increased tolerance to applications of glyphosate and sulfometuron methyl (Michler and Haissig, 1988). Disease tolerance to Septoria leaf spot was increased in hybrid poplar (Ostry and Skilling, 1988) and to $M e$ lampsora leaf rust in cottonwood (Prakash and Thielges, 1989). Plants with stable leaf variegation were regenerated to produce periclinal chimeras of sweetgum (Brand and Lineberger, 1988) and irregular variegation of hybrid poplar (unpublished data).

Agrobacterium -mediated gene insertion techniques have been used to transform hybrid poplars to produce trees with commercially important introduced genes. Fillatti et al. (1987) introduced the aroA gene that confers resistance to glyphosate. Gene insertion and expression were confirmed, but commercial levels of resistance have not yet been produced (Riemenschneider et al., 1988). Research is continuing to improve levels of resistance by altering promoter sequences (D. Riemenschneider, personal communication). Klopfenstein et al. (1989) introduced into three hybrid poplar clones a gene for insect resistance that contained a wound inducible promoter controlling proteinase activity, and gene insertion was confirmed in regenerated plants. Leaf bioassays confirmed increased enzyme expression resulting from the introduced gene.

Other gene insertion techniques have been successfully applied to forest tree species and confirmed by bioassay and histochemical tests for inserted marker genes. Biolistics was used to insert DNAcoated gold particles into hybrid poplar protoplast-derived cells, 
internodes, and nodules, and white spruce embryogenic callus and zygotic embryos (Ellis et al., 1989). Transformation was confirmed by transient expression of inserted B-glucuronidase genes (GUS), and transformed hybrid poplar plants with the inserted GUS gene were regenerated. Gupta et al. (1988) electroporated protoplasts of Douglas fir and loblolly pine and inserted DNA, which contained a marker gene for luciferase. Others have used electroporation techniques to introduce GUS genes into Alnus incana protoplasts (Sequin and Lalonde, 1988) and GUS and chloramphenicol acetyltransferase (CAT) genes into Picea glauca protoplasts (Bekkaoui et al., 1988). Wilson et al. (1989) used PEG-mediated DNA insertion to deliver GUS and CAT genes into protoplasts from embryogenic cell suspension callus of Picea glauca.

\section{PURPOSES OF BIOTECHNOLOGY WITH WOODY ENVIRONMENTAL CROPS}

The following areas of woody environmental plant improvement are likely targets, despite the lack of current research: General areas of genetic improvement include plant resistance to biotic and environmental stresses (which can increase and broaden edaphic adaptability), improvement of ornamental phenology and plant morphology, and several other characters.

Many economically important woody environmental plants are sensitive to the stress of biotic agents, environmental irritants, and non-native soil characteristics. These stresses can result from insect and disease infestations, drought, herbicides, salt, incompatible soil type and structure, toxic metals, frost, temperature extremes, air pollutants, and mechanical wounding. Exposure to such stresses limits the long-term fitness of woody ornamentals in the landscape.

Ornamental characters that could be improved involve alteration of morphology or phenology. Some examples are the size, shape, and color of leaves, fruits, and flowers; overall plant shape, such as dwarfness and altered branch angle; and bark color and exfoliation traits. Changes in such characters would improve visual attractiveness and extend the usage of these improved plants in many smaller or difficult landscape sites.

Other traits and characteristics that would improve the economic value of woody environmental plants are altered or extended flowering time, rootstock characteristics, and production of secondary products. Some plants are limited in their usage because of flowering time, and delay or extension could extend the plant's utility in some zones. Performance of plants that are budded or grafted onto seedling or nonclonal rootstocks could be improved by selection of rootstocks for characteristics that would enhance the fitness of scion material. Finally, secondary product formation from woody environmental plants is virtually unstudied but might result in substantial added value.

\section{PRESENT STATUS AND CONSTRAINTS OF BIOTECHNOLOGY}

There are four reasons for limited use of biotechnology with woody environmental plants to date; namely, lack of: 1) adequate in vitro regeneration techniques for many species, 2) interest by growers and user groups, 3) funding from granting agencies and industry, and 4) trained scientists who can apply these techniques.

In vitro regeneration techniques that can be used in genetic engineering applications are still lacking for many species. However, this problem is being solved, and the literature contains scattered reports of successful regeneration of woody environmental plants from protoplasts, cell suspension callus, and leaf explants. Regeneration of woody environmental plants from adult tissue is still difficult and is an area for continued research.

Growers and consumers generally have lacked interest in genetically improved woody environmental plants because of the many cultivars available to them. Research to produce improved plants has not been initiated because of the lack of markets and funding.

Industry and granting agencies have given biotechnology of woody omamentals a low priority for funding. Industry has provided small grants for micropropagation research, but has been unwilling to make long-term commitments to genetic engineering research that sometimes requires costly equipment and extended evaluation of genetically improved plants in the greenhouse and field. Granting agencies have limited funds, and woody ornamental plant improvement does not take national priority over many other problems related to food crops and the environment. Scientists who are actively involved in biotechnology research have sometimes funded it with monies received for other general purposes.

Few scientists who work with woody environmental plants are trained in biotechnology, primarily for two reasons: 1) lack of retraining older scientists and, 2) lack of hiring young scientists trained in this area of expertise. Many older scientists have realized that biotechnology training is needed and are beginning to seek this knowledge through sabbatical leaves and training courses. Young, recently trained scientists will be hired only if administrators are convinced of the long-term need and if candidates are available.

\section{FUTURE DIRECTIONS}

Many woody environmental plant problems could be targeted for plant improvement research programs that use available genes and biotechnology techniques. Only a few of the many opportunities will be outlined in this discussion.

Using in vitro genetic selection, we can increase herbicide tolerance in many woody environmental species that are subjected to broad-spectrum herbicides, such as glyphosate, sulfometuron methyl, and bialaphos. Tolerance to these herbicides could reduce plant damage and loss encountered during nursery production and landscape maintenance. Resistance to leaf spot and scab diseases of ornamental crabapple could be increased by using technology developed by Hammerschlag and Ognjanov (1989) for peach. Also, many woody environmental plants are too large for use in small landscapes, and selected genetic variants with more compact growth habits could be useful for these landscapes.

Using gene insertion techniques, we might enhance resistance to bronze birch borer and Japanese beetle by inserting Bacillus thurengiensis (BT) or proteinase genes into birch and rose. Furthermore, disease resistance of rose to powdery mildew might be enhanced by insertion of chitinase genes.

Future needs must be met to take full advantage of opportunities for use of biotechnology in woody environmental plant improvement. First, research programs need to be established that have woody environmental plant improvement as the main focus. Second, we must improve regeneration techniques and then work with molecular biologists to use desirable genes that they have isolated and use their knowledge of genomic mapping to aid breeding. Third, micropropagation costs need to be reduced through automation; advancement may be forthcoming in this area. Fourth, we need a greater basic understanding of tree physiology to solve problems of regeneration and epigenetic effects that plague the use of some woody environmental species originated in vitro. Fifth, we must educate growers and consumers to the attributes of genetically engineered plants. Many of our improved woody environmental plants will result in reduced plant maintenance, pesticide usage, and plant loss. In. the end, the consumer must be willing to pay more for these products because of the added value. Finally, we need to fully substantiate products of our biotechnological manipulations to ensure genetic stability of altered plant genomes. This goal may require the use of serial vegetative propagation, biochemical and molecular analysis, extensive field testing, and breeding, the latter for evaluation and introduction of novel genes into important germplasm lines.

\section{SUMMARY}

Biotechnology of woody environmental plants awaits development. Such development will depend on financial support and will borrow directly from plant improvement techniques already being applied to forest and fruit trees. Furthermore, we must commit ourselves fully to this research, develop needed skills, educate users, and promote the use of these value-added products.

\section{Literature Cited}

Bekkaoui, F., M. Pilon, E. Laine, D.S.S. Raju, W.L. Crosby, and D.I. Dunstan. 1988. Transient gene expression in electroporated Picea glauca protoplasts. Plant Cell Rpt. 7:481-484. 
Brand, M.H. and R.D. Lineberger. 1988. In vitro adventitious shoot formation on mature-chase leaves and petioles of Liquidambar styraciflua L. Plant Sci. 57:3173-179.

Ellis, D.D., B.H. McCown, J.A. Russell, J.C. Sellmer, and D.E. McCabe. 1989. Genetic transformation of trees: The introduction of foreign genes via electrical discharge particle acceleration. Proc. XIX Stadler Genet. Symp. on Gene Manipulation in Plant Improvement II, Univ. of Missouri-Columbia. 13-15 Mar. 1989. (Abstr.).

Fillati, J.J., J. Sellmer, B. McCown, B. Haissig, and L. Comai. 1987. Agrobacterium -mediated transformation and regeneration of Populus. Mol. Gen. Genet. 206:192-199.

Gupta, P.K., A.M. Dandekar, and D.J. Durzan. 1988. Somatic proembryo formation and transient expression of a luciferase gene in Douglas fir and Loblolly pine protoplasts. Plant Sci. 58:85-92.

Hammerschlae. F.A. and V. Ognjanov. 1989. Somaclonal variation in peach: Screening for resistance to Xanthomonas campestris pv. pruni and Pseudomonas syringae pv. syringae. Acta Hort. 280:403-408.

Klopfenstein, N.B., S.A. Heuchelin, H.S. McNabb, Jr., R.W. Thornburg, R.B. Hall, and E.R. Hart. 1989. Transformation of hybrid Populus with the proteinase inhibitor II gene, Phytopathology 79(9):1004.

Larkin, P. and W. Scowcroft. 1981. Somaclonal variation-A novel source of variability from cell cultures for plant improvement. Theor. Appl. Genet. 60:197-214.
Marcotrigiano. M. and L. Jagannathan. 1988. Paulownia tomentosa 'Somaclonal Snowstorm'. HortScience 23:226-227.

McCown, B.H. 1989. The biotechnology of urban trees. J. Arboriculture 15(4):77-83.

Michler, C.H. and B.E. Haissig. 1988. Increased herbicide tolerance of in vitro selected hybrid poplar, D. 183-189. In: M.R. Ahuja (ed.). Somatic cell genetics woody plants. Kluwer Academic Publishers, Dordrecht. The Netherlands.

Ostry, M.E. and D.D. Skilling. 1988. Somatic variation in resistance of Populus to Septoria musiva. Plant Dis. 72:724-727.

Prakash, C.S. and B.A. Thielges. 1989. Somaclonal variation in eastern cottonwood for race-specific partial resistance to leaf rust disease. Phytopathology 79:805-808.

Riemenschneider, D.E., B.E. Haissig, J. Sellmer, and J.J. Fillatti. 1988. Expression of an herbicide tolerance gene in young plants of a transgenic hybrid poplar clone, p. 73-80. In: M.R. Ahuja (ed.). Somatic cell genetics of woody plants. Kluwer Academic Publishers, Dordrecht, The Netherlands.

Sequin, A. and M. Lalonde. 1988. Gene transfer by electroporation in betulaceae protoplasts: Alnus incana. Plant Cell Rot. 7:367-370.

Wilson, S.M, T.A. Thorpe, and M.M. Maloney. 1989. PEG-mediated expression of GUS and CAT genes in protoplasts from embryogenic suspension cultures of Picea glauca. Plant Cell Rpt. 7:704-707. 